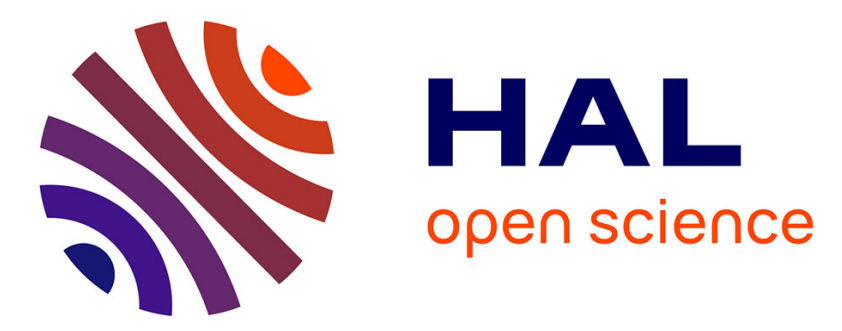

\title{
An Optimization Approach for the Synthesis of AUTOSAR Architectures
}

Ernest Wozniak, Asma Mehiaoui, Chokri Mraidha, Sara Tucci-Piergiovanni, Sébastien Gerard

\section{- To cite this version:}

Ernest Wozniak, Asma Mehiaoui, Chokri Mraidha, Sara Tucci-Piergiovanni, Sébastien Gerard. An Optimization Approach for the Synthesis of AUTOSAR Architectures. Emerging Technologies \& Factory Automation (ETFA), 2013 IEEE 18th Conference on, Sep 2013, Cagliari, Italy. cea-01810019

HAL Id: cea-01810019 https://hal-cea.archives-ouvertes.fr/cea-01810019

Submitted on 7 Jun 2018

HAL is a multi-disciplinary open access archive for the deposit and dissemination of scientific research documents, whether they are published or not. The documents may come from teaching and research institutions in France or abroad, or from public or private research centers.
L'archive ouverte pluridisciplinaire HAL, est destinée au dépôt et à la diffusion de documents scientifiques de niveau recherche, publiés ou non, émanant des établissements d'enseignement et de recherche français ou étrangers, des laboratoires publics ou privés. 


\title{
An Optimization Approach for the Synthesis of AUTOSAR Architectures
}

\author{
Ernest Wozniak, Asma Mehiaoui, Chokri Mraidha, Sara Tucci-Piergiovanni and Sébastien Gerard \\ CEA, LIST, Laboratory of Model-Driven Engineering Applied to Embedded Systems \\ 91191 Gif-sur-Yvette CEDEX, FRANCE \\ \{name.lastname\}@cea.fr
}

\begin{abstract}
Synthesis of automotive architectures is a complex problem that needs an automated support. AUTOSAR, standard for the specification of automotive architectures, defines a synthesis process of software components and their connections in a set of fixedpriority OS tasks distributed over a network of ECUs. During the synthesis process software components are allocated on ECU-s. Since each component encapsulates a set of so-called runnable entities, synthesis completes by partitioning runnable entities in OS tasks with assigned fixed priorities. This paper proposes an optimization approach for the synthesis of AUTOSAR architectures based on genetic algorithms and mixed integer linear programming techniques. Optimization criteria consider end-to-end timing responses and memory consumption.
\end{abstract}

\section{Introduction}

The AUTOSAR (AUTomotive Open System Architecture) [1] development process aligns to the MDE (Model Driven Engineering) principles as it is based on the use of models defined through metamodels and provides a development process based on a progressive refinement of models. At the heart of the development process lays the synthesis of the functional architecture (encapsulating the system logic) in a real-time architecture made of fixed-priority tasks distributed over a network of ECU-s. The synthesis process according to the AUTOSAR can be divided in four main activities. The first one is called the allocation as it consists in placing the atomic software components on the ECU-s and the exchanged signals on the buses. Each software component encapsulates the implementation of a specific functionality which is exposed to the outside world by means of ports. Internal behaviour of each atomic software component is represented by a graph of runnable entities, which in turn represent schedulable units of computation. Let us remark that each atomic software component contains at least one runnable entity. Runnables of the same component cannot be split among different ECU-s, hence we can say that allocation of atomic software components determines the allocation of runnables.
From now on, when discussing allocation we refer to the allocation of runnable entities and data signals.

Second, third and fourth activity are partitioning, scheduling and ordering. Runnables and signals are being partitioned in OS tasks and messages which are then scheduled by the assignment of static priorities. Moreover the order of runnables inside a task is defined with respect to the functional constraints.

It is common and recommended practice [2] in this demarche, to specify the end-to-end timing constraints at the highest level, between input and output ports of the highest level component of the architecture, usually representing the system under study (see Figure 1). For each external stimulus, consumed by an input port, the constraint specifies a deadline for the response to be produced on the output port. Each end-to-end constraint is progressively refined by specifying the end-to-end chain of runnable entities (traversing one or more atomic components) that are activated to produce a system response triggered by the given stimulus.

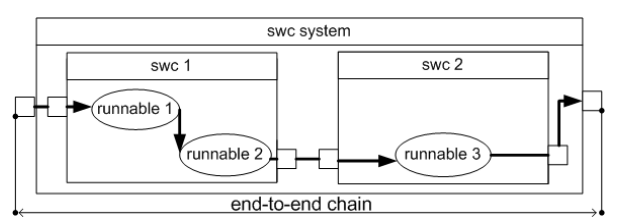

Figure 1 Visualization of end-to-end chain

By knowing runnables allocation their partitioning and order within the tasks as well as priorities of the tasks, the designer can now compute the response times of runnables end-to-end chains to see if end-toend timing constraints are met. If some deadlines are violated, the designer has to find another configuration. This is a cumbersome process as the synthesis is an NP-hard problem. Hence appropriate support is required. In the current state of practice, only partial solutions exist as none of them handle all four dimensions (allocation, partitioning scheduling and ordering) at once. Most common solutions take as input a task model which means that the partitioning is already known or is done manually based on an engineer's expertise. This severely minimizes the design space to explore and therefore might exclude feasible solutions as shown later in this work.

This paper presents two techniques for the synthesis of AUTOSAR architectures in its entire form. The first 
technique is based on mixed integer linear programming (MILP). It returns the optimal solution but is limited to small size systems. In order to improve scalability - to address industrial size systems - a second technique, based on the genetic algorithms (GA) is proposed. For both techniques optimization criteria relate to the end-to-end responses and consumed memory. As runnables on the same ECUs communicate asynchronously through shared variables, a protection mechanism is required to guarantee data consistency and behaviour predictability. Protection mechanisms either take time or consume memory. Our techniques will operate a choice between a timeconsuming and a memory-consuming protection mechanism, when optimizing response time and memory consumption.

This paper is organized as follows. The next section presents related work. Section 3 formalizes the system model, presents used schedulability analysis and memory consumption analysis, i.e. the way in which end-to-end responses and memory overhead are computed. Finally this section formulates the synthesis problem and optimization criteria. Section 4 presents the optimization technique, i.e. the Genetic Algorithms and the MILP formulation. Section 5 evaluates our techniques. First, results of the GA technique are compared against the MILP results. Then the GA technique is evaluated against current approaches disregarding the partitioning dimension. Section 6 concludes the paper and discusses the future work.

\section{Related Work}

The literature on the synthesis problem is rich. There are many approaches that consider only the allocation and task scheduling problems and relate to different optimization metrics (like the inter-ECU communication bandwidth) [3-6].

Concerning the system model and the optimization objectives our approach is closely related to the following works [7-12]. The [7] and [8] similarly as in our approach consider periodic activation and end-toend responses as optimization criteria. The main difference is that the authors are considering OS tasks as an allocation unit and hence partitioning and ordering is fixed for them. Ferrari et al. [9] is the first work discussing possible strategies to protect shared data items and memory/timing tradeoffs. The work in [10] proposes a two-step technique for the allocation of AUTOSAR software components to the ECUs, taking into account protection mechanism as a parameter to specify. However it considers neither partitioning nor ordering. Authors of [11] and [12] also relate to the periodic runnables in their model. They consider additional mechanisms that can assure data consistency like the absence of preemption. The last can be done by defining so called preemption thresholds or preemption groups. In their work the allocation is fixed and hence their approach is for local optimization. Interestingly, the order of runnables as the parameter to manipulate is considered.

The main contribution of our work in relation to the current approaches ([13] gives an exhaustive survey on the existing methods for the optimization of real-time embedded systems) lies in a holistic deployment of the automotive architectures. None of the existing works is solving the four problems, i.e. allocation, partitioning, scheduling and ordering together, considering also assignment of a memory protection mechanism.

\section{Formalism}

\subsection{System Model}

The input system model consists of two graphs. The first one is an AUTOSAR execution model represented by a directed graph $G_{e}=\left\{V_{e}, E_{e}\right\}$ in which $V_{e}$ is the set of vertices representing runnables and $E_{e}$ is the set of edges related to the links between them. The second one is an undirected graph $G_{h}=\left\{V_{h}, E_{h}\right\}$ that expresses hardware architecture. Nodes represent hardware resources and the edges represent communication links between them. The hardware resources are ECUs and communication buses. The remaining notions used throughout this work were gathered in the table below for a better readability.

\begin{tabular}{|c|c|}
\hline Concep & Explanation \\
\hline$E$ & Set of ECUs \\
\hline$e_{i}$ & ECU \\
\hline$\beta$ & Set of BUSes \\
\hline$b_{i}$ & BUS \\
\hline$E\left(b_{i}\right)$ & $\begin{array}{l}\text { This function returns set of the ECUs } \\
\text { communicating through the bus } b_{i}\end{array}$ \\
\hline$R$ & $\begin{array}{l}\text { Set of runnable entities. In this work we restrict to } \\
\text { periodic runnables, i.e. runnables that are activated } \\
\text { in response to periodic timer events. }\end{array}$ \\
\hline$r_{i}$ & Runnable entity \\
\hline$P_{r_{i}}$ & Period of a runnable entity \\
\hline$d_{r_{i}}$ & Local deadline of the runnable entity $r_{i}$ \\
\hline $\begin{array}{c}\vec{C}_{r_{i}}= \\
\left(C_{r_{i}, e_{1}}\right. \\
C_{r_{i}, e_{2}} \\
\left.C_{r_{i}, e_{n}}\right) \\
\end{array}$ & $\begin{array}{l}\text { Worst case execution time of a runnable, } \\
\text { characterized by a vector of WCETs, due to the } \\
\text { heterogeneity of the hardware nodes. Later in the } \\
\text { paper we omit the second index specifying the ECU, } \\
\text { just for the simplicity of the notation. }\end{array}$ \\
\hline$s w c_{i}$ & $\begin{array}{l}\text { Atomic software component. Its behaviour is } \\
\text { defined by the runnable entities. }\end{array}$ \\
\hline$S C\left(r_{i}\right)$ & $\begin{array}{l}\text { This function returns atomic software component of } \\
\text { a runnable } r_{i} \text {. }\end{array}$ \\
\hline$P S_{r_{i}}$ & Communication ports of runnable $r_{i}$ \\
\hline$P_{r_{i}}^{\text {in }}$ & Set of input ports of the runnable entity $r_{i}$ \\
\hline$p_{r_{i}, i}^{i n}$ & $j^{t h}$ input port of the runnable $r_{i}$ \\
\hline$P_{r_{i}}^{\text {out }}$ & Set of output ports of the runnable entity $r_{i}$ \\
\hline$p_{r_{i}, i}^{\text {out }}$ & $j^{t h}$ output port of the runnable $r_{i}$ \\
\hline
\end{tabular}




\begin{tabular}{|c|c|}
\hline$S$ & et of data signals \\
\hline$s_{i}{ }^{1}$ & Data signal \\
\hline$D S\left(s_{i}\right)$ & Size of the data signal $s_{i}$ \\
\hline$\Omega$ & Set of shared resources \\
\hline$\sigma_{i}$ & Shared resource \\
\hline$r^{w}\left(\sigma_{i}\right)$ & $\begin{array}{l}\text { Set of writer runnables, writing to the shared } \\
\text { resource } \sigma_{i} \text {. We consider one-to-many } \\
\text { communication hence } r^{w}\left(\sigma_{i}\right) \text { contains only one } \\
\text { writer. }\end{array}$ \\
\hline$r^{r}\left(\sigma_{i}\right)$ & Set of readers of the shared resource $\sigma_{i}$ \\
\hline$\zeta\left(\sigma_{i}\right)$ & Data signal of the shared resource $\sigma_{i}$ \\
\hline$\zeta^{\prime}\left(s_{i}\right)$ & Shared resource corresponding to the signal $s_{i}$ \\
\hline$\Omega_{r_{i}}$ & $\begin{array}{l}\text { Set of all the shared resources accessed by the } \\
\text { input/output ports of the runnable } r_{i}\end{array}$ \\
\hline $\begin{array}{l}\omega_{p_{r_{i}, j}^{\text {in }}} \\
/ \omega_{p_{r_{i}, k}^{\text {out }}}\end{array}$ & $\begin{array}{l}\text { WCET of the runnable entity } r_{i} \text { on the critical } \\
\text { section used for accessing shared resources. The } \\
\text { access is through the input/output port } p_{r_{i}, i}^{\text {in }} / p_{r_{i}, k}^{\text {out }}\end{array}$ \\
\hline $\begin{array}{l}\xi= \\
\left\{\Gamma_{1}\right. \\
\Gamma_{2} \\
\cdots, \\
\left.\Gamma_{n}\right\}\end{array}$ & $\begin{array}{l}\text { Set of the end-to-end chains that we call paths. Each } \\
\text { path is defined as an ordered interleaving sequence } \\
\text { of runnables and signals defined as } \Gamma_{i}= \\
{\left[r_{o_{1}}, s_{O_{1}}, r_{O_{2}}, s_{O_{2}}, \ldots, s_{O_{k-1}}, r_{o_{k}}\right] . \operatorname{src}\left(\Gamma_{i}\right)=r_{o_{1}} \text { is the }} \\
\text { path's source and } \operatorname{snk}\left(\Gamma_{i}\right)=r_{o_{k}} \text { is the sink. Multiple } \\
\text { paths may exist between each pair of source-sink. }\end{array}$ \\
\hline $\mathrm{R}_{\Gamma_{j}}$ & e time of the path $\Gamma_{i}$ \\
\hline $\mathrm{D}_{\Gamma_{i}}$ & Deadline of the path $\Gamma_{i}$ \\
\hline$\tau_{i}$ & $\begin{array}{l}\text { of a runnable entity executes in a } \\
\text { sk }\end{array}$ \\
\hline$e\left(\tau_{i}\right)$ & ECU on which th \\
\hline$e\left(r_{i}\right)$ & the runnable $r_{i}$ is allocated \\
\hline$e\left(\sigma_{i}\right)$ & ECU on which shared resource $\sigma_{i}$ is specified \\
\hline$\tau\left(r_{i}\right)$ & Task i \\
\hline$i d x\left(r_{i}\right)$ & $\begin{array}{l}\text { As multiple runnables can be partitioned in one task } \\
\text { we specify the order by introducing the index of a } \\
\text { runnable inside a task } j=i d x\left(r_{i}\right) \text {. Hence } r_{i, j} \text { means } \\
\text { that runnable } r_{i} \text { is at the } j^{t h} \text { position in a task. }\end{array}$ \\
\hline$\pi_{r_{i}}$ & $\begin{array}{l}\text { Priority of a runnable entity. It equals to the priority } \\
\text { of } \tau\left(r_{i}\right) \text { which is defined as } \pi_{\tau}\end{array}$ \\
\hline$C_{\tau_{i}}$ & $\begin{array}{l}\text { WCET of the task } \tau_{i} \text {. It equals to the sum of } \\
\text { WCETs of all the runnables partitioned within this } \\
\text { task, i.e. } C_{\tau_{i}}=\sum_{\tau\left(r_{i}\right)=\tau_{i}} C_{r_{i}} \text {. }\end{array}$ \\
\hline$m_{k}$ & BUS message \\
\hline$m\left(s_{i}\right)$ & Message that transmits the signal $s_{i}$ \\
\hline \begin{tabular}{c|}
$\vec{C}_{S_{i}}=$ \\
$\left(C_{S_{i}, b_{1}}\right.$ \\
$C_{S_{i}, b_{2}}$ \\
$\quad \ldots$ \\
$\left.C_{S_{i}, b_{n}}\right)$
\end{tabular} & $\begin{array}{l}\text { WCTT (Worst Case Transmission Time) of the } \\
\text { signal } s_{i} \text { when transmitted on the BUS. Also here } \\
\text { for the sake of simplicity, the second index } \\
\text { indicating the bus is omitted when relating to the } \\
\text { signal WCTT. }\end{array}$ \\
\hline$C_{m_{i}}$ & WCTT of the message $m_{i}$. It is a sum of WCTT of \\
\hline
\end{tabular}

1 Runnables communicate by sharing data signals accessed through their ports. Data signal can be communicated either through a shared resource or via a message passing. The identification of shared resources uniquely depends on the runnables allocation and partitioning. For each identified data signal communicated between runnables of different tasks but of the same ECU we define a shared resource. Data signals communicated between runnables of the same task don't require defining a shared resource. Also, for the inter-ECU communication no shared resource is required as in this case data signal is communicated by the BUS message.

\begin{tabular}{|l|l|}
\hline & $\begin{array}{l}\text { all the signals partitioned in this message, i.e. } \\
C_{m_{i}}=\sum_{m}\left(s_{i}\right)=m\end{array} . C_{S_{i}}$. \\
\hline$P_{s_{i}}$ & $\begin{array}{l}\text { Period of the signal } s_{i} . \text { It is equal to the period of a } \\
\text { writer runnable. }\end{array}$ \\
\hline
\end{tabular}

Table 1. Main Concepts of the System Model

\subsection{Memory}

The manner in which allocation and partitioning of runnables is done has an impact on the used memory. First, as it is specified in the AUTOSAR metamodel [14], runnable entity $r_{i}$ has a stack memory usage that differs, depending on the ECU on which it is deployed. This is specified with the vector $\vec{M}_{r_{i}}=\left(M_{r_{i}, e_{1}}, M_{r_{i}, e_{2}}, \ldots, M_{r_{i}, e_{n}}\right)$. The next factor affecting the used memory is the protection mechanism specified to protect the shared resources. Protection mechanism has to be specified for each signal communicated between runnables of different tasks that are deployed on the same ECU. This is due to the asynchronous communication between periodic runnables and hence, mechanism to provide the data consistency is necessary. This work considers two mechanisms:

- Wait-free access method such as Rate Transition (RT) block [15] - this mechanism behaves like a Zero-Order Hold block or a Unit Delay block plus a Hold block or Sample and Hold (for slow to fast transitions). Its implementation consists of a switched buffer. This mechanism incurs negligible time overhead but it consumes additional memory.

- $\quad$ Semaphore Lock (SL) - in this work we assume immediate priority ceiling semaphores. Priority of a runnable that is accessing a shared resource is raised to the ceiling priority of a resource. The $\mathrm{SL}$, opposite to the RT, imposes no additional memory overhead, however it suffers timing delays in the form of a blocking time.

Function $\gamma\left(\sigma_{i}\right)$ will return the value representing the protection mechanism used to protect a shared resource $\sigma_{i}$. Value $S L$ concerns semaphore lock, whereas $R T$ means Rate Transition block. Overall memory overhead $M_{e_{i}}$ for ECU $e_{i}$ is computed according to (1).

$$
M_{e_{i}}=\sum_{e\left(r_{j}\right)=e_{i}} M_{r_{j}}+\sum_{e\left(\sigma_{k}\right)=e_{i}} M_{\sigma_{k}}
$$

The $M_{\sigma_{k}}$ is a memory overhead caused by the RT and is computed according to [11] (see (2)). For this we define additional notation. We denote the set of readers with higher (lower) priority than the writer $r^{w}\left(\sigma_{i}\right)$ as $r^{H R}\left(\sigma_{i}\right) \quad\left(r^{L R}\left(\sigma_{i}\right)\right)$. Our formula is a simplification of what is included in [11] as in this work we are not considering the preemption thresholds.

$$
M_{\sigma_{k}}=D S\left(\zeta\left(\sigma_{k}\right)\right) n_{k}
$$




$$
\begin{gathered}
n_{\sigma_{k}}=\left\{\begin{array}{c}
\sum_{r_{i} \in r^{w}\left(\sigma_{k}\right), r_{j} \in r^{L R}\left(\sigma_{k}\right)} w_{k}+2 w_{k} \text { ifr } r^{H R}\left(\sigma_{k}\right) \neq \varnothing \\
\sum_{r_{i} \in r^{w}\left(\sigma_{k}\right), r_{j} \in r^{L R}\left(\sigma_{k}\right)} w_{k}+w_{k} \text { ifr } r^{H R}\left(\sigma_{k}\right)=\emptyset
\end{array}\right. \\
w_{\sigma_{k}}=\left\{\begin{array}{l}
1 \text { if } \gamma\left(\sigma_{i}\right)=R T \\
0 \text { if } \gamma\left(\sigma_{i}\right)=S L
\end{array}\right.
\end{gathered}
$$

\subsection{Timing Analysis}

Timing analysis concerns computation of the response times for runnables and global signals and also computation of the end-to-end responses. It is based on the work presented in [8] and adapted to consider runnable entities. Adaptation is because the entities considered in the analysis of [8] focus on the OS tasks and doesn't consider functional architecture as in our case.

\subsubsection{Schedulability of Runnable Entities}

Worst case response time of runnable $r_{i}$, for which $i d x\left(r_{i}\right)=j$, is represented with $R_{r_{i, j}}$ and computed according to (5). The $C_{\tau_{i, j}}$ (see eq. 6 ) is the worst case computation time of the task until the $j^{\text {th }}$ runnable partitioned in this task. Please note that we allow partitioning of the runnables with the harmonic periods in the same task. This means that when the task is executed not all of the runnables will be activated. Therefore the $C_{\tau_{i, j}}$ varies. However we assume the worst case scenario hence we account for all the runnables up till the $j^{\text {th }}$ when computing the $C_{\tau_{i, j}}$. The $B_{\tau_{i}}$ is a blocking time of a task $\tau_{i}$. Blocking time depends on the shared resources accessed by the task and the way in which the shared resources are protected from multiple accesses. If the shared resource is protected with a semaphore lock, it causes a blocking time. The semaphore lock in our case is realized through the Priority Ceiling Protocol (PCP) [16]. The same blocking time applies to all the runnables that are partitioned in the same task and therefore it is computed for a task. To compute the blocking time with the PCP few additional things have to be clarified. First, the shared resources of a task $\tau_{i}$ are specified with the set $\Omega_{\tau_{i}}=\cup_{\tau\left(r_{j}\right)=\tau} \Omega_{r_{j}}$. This means that the task inherits the access to the shared resources from the runnables partitioned in this task. The WCET of a task $\tau_{i}$ for accessing (reading/writing) a critical section of a shared resource $\sigma_{i}$ is represented with $\tau_{i}\left(\omega_{\sigma_{i}}^{\text {in }}\right)=$ $\max _{r_{i}}\left(\omega_{p_{r_{i}, j}^{\text {in }}}\right) / \tau_{i}\left(\omega_{\sigma_{i}}^{\text {in }}\right)=\max _{r_{i}}\left(\omega_{p_{r_{i j}}^{\text {out }}}\right)$. Function $h p\left(r_{i}\right)$ returns all the runnable entities allocated on the same ECU as $r_{i}$, with the priority higher than $r_{i}$.

$$
\begin{gathered}
R_{r_{i, j}}=B_{\tau\left(r_{i}\right)}+C_{\tau\left(r_{i}\right), j}+\sum_{r_{k} \in h p\left(r_{i}\right)}\left[\frac{R_{r_{i, j}}}{P_{r_{k}}}\right] C_{r_{k}} \\
C_{\tau_{i, j}}=\sum_{\tau\left(r_{k}\right)=\tau_{i} \wedge k \leq j} C_{r_{k}}
\end{gathered}
$$

\subsubsection{Schedulability of Signals}

Worst case response time for a signal is computed in case when $s_{i}$ represents inter-ECU communication.
We are considering an event triggered bus, such as the CAN bus. Similarly to (5) the computation of $R_{S_{i}}$ accounts for a blocking time $B_{b_{l}}$. This blocking time characterizes entire bus $b_{l}$. It is caused due to the nonpreemptive scheduler of a bus, such as the CAN. This blocking time $B_{b_{l}}$ applies to all the signals of a bus $b_{l}$, except those partitioned in a message with the lowest priority. Function $h p\left(m_{i}\right)$ returns all the messages of the same bus as $m_{i}$ with a priority higher.

$$
R_{s_{i}}=B_{b_{l}}+C_{m\left(s_{i}\right)}+\sum_{m_{k} \in h p\left(m\left(s_{i}\right)\right)}\left\lceil\frac{R_{s_{i}}}{P_{S_{i}}}\right\rceil C_{m_{k}}(7)
$$

\subsubsection{End-to-end Responses Computation}

The worst case end-to-end latency $R_{\Gamma_{i}}$ is computed for each path $\Gamma_{i}$ by adding the worst case response times of all the runnables and global signals (i.e. signals representing intra-ECU communication), as well as the periods of all the global signals and their reader runnables on the path (see (8)). Set $\Phi$ represents all the global signals. The $\operatorname{read}_{s_{k}, \Gamma_{i}}$ is the reader runnable of $s_{k}$ on the specific path $\Gamma_{i}$.

$$
R_{\Gamma_{i}}=\sum_{\mathrm{r}_{j} \in \Gamma_{i}} R_{\mathrm{r}_{j}}+\sum_{\mathrm{s}_{k} \in \Gamma_{i} \wedge s_{k} \in \Phi} R_{\mathrm{s}_{k}}+P_{s_{k}}+P_{\text {read }_{s_{k}, \Gamma_{i}}}
$$

\subsection{Problem Formulation}

The main objective of this work is an automated support for the synthesis problem, i.e., the integration of functional architecture (represented by the communicating, periodic runnable entities and the exchanged data signals) with the execution platform, i.e. ECUs/BUSes and OS tasks/messages. This means that for each runnable/data signal, its hosting ECU/BUS has to be assigned (allocation), then runnables/data signals allocated to the same ECU/BUS have to be partitioned in tasks/messages, for which the priorities needs to be defined. Also the order of runnables inside the task needs to be established as it has a significant impact on the runnables local responses and in consequence end-to-end responses as well. Additionally, to protect the shared resources the protection mechanism needs to be specified (either RT or SL). This process should also respect multiple constraints which are specified with our MILP formulation (see subsection 4.2). Finally and most importantly, the synthesis process is driven by predefined optimization criteria. We are defining four optimization metrics and for each, its importance can be specified by assigning a weight. Therefore our final fitness function $F\left(\Psi_{i}\right)$ where $\Psi_{i}$ represents a final configuration, i.e. deployed architecture, is a weighted sum of four functions: $F\left(\Psi_{i}\right)=w_{1} \mathrm{f}_{e 2 e}\left(\Psi_{i}\right)+$ $w_{2} \mathrm{f}_{b_{m}}\left(\Psi_{i}\right)+w_{3} \mathrm{f}_{t h}\left(\Psi_{i}\right)+w_{4} \mathrm{f}_{r_{l d}}\left(\Psi_{i}\right)$ explained below.

1) End-to-end Responses Optimization

Optimization of the end-to-end responses (9) aims at minimizing the response times of paths, relatively to their deadlines. Their optimization improves the system performance. 


$$
\mathrm{f}_{e 2 e}\left(\Psi_{i}\right)=1-\sum_{\Gamma_{j}} \frac{R_{\Gamma_{j}}}{\mathrm{D}_{\Gamma_{j}}}
$$

\section{2) Memory Optimization}

Optimization of memory aims at minimizing the additional memory overhead that can be caused by using the Rate Transition blocks and inappropriate balancing when placing runnables on the ECUs. The last is due to the heterogeneous nature of the ECUs.

$$
\mathrm{f}_{m}\left(\Psi_{i}\right)=|E|-\sum_{e_{j}} \frac{M_{e_{j}}}{M_{e_{j}}^{\text {max }}}
$$

The $M_{e_{i}}^{\max }$ represents the worst case possible memory overhead caused for $e_{i} ; M_{e_{i}}^{\max }=$ $\sum_{r_{j}} M_{r_{j}, e_{i}}+\sum_{\sigma_{k}} M_{\sigma_{k}}$. Its computation assumes that each runnable is partitioned in one task, writer has always higher priority than all its readers and all shared resources are protected with RT.

3) Bus Throughput

This objective concerns the increase of the buses throughput. For this (11) is used. Optimization of throughput is a common approach to provide higher extensibility of a bus.

$$
\mathrm{f}_{b_{t h}}\left(\Psi_{i}\right)=1-\frac{\sum_{s_{j} \in \Phi} S\left(\mathrm{~s}_{j}\right)}{{\sum \mathrm{s}_{k} \in \mathrm{S}}_{\mathrm{s}_{k}} S\left(\mathrm{~s}_{k}\right)}
$$

\section{4) Runnables Local Deadlines}

The runnables local deadlines optimization aims at minimizing the response times of runnables, relatively to their local deadlines (if any). Local deadlines might result from the refinement of the end-to-end timing constraints.

$$
\mathrm{f}_{r_{l d}}\left(\Psi_{i}\right)=1-\sum_{r_{j}} \frac{R_{\mathrm{r}}}{\mathrm{D}_{r_{j}}}
$$

\section{Optimization Technique}

This section presents the Genetic Algorithm and the MILP formulation used to solve the synthesis problem. The MILP assures provision of an optimal solution if the solver terminates with no error. This property makes it a good comparator for such heuristic approach as the GA. The shortcoming of the MILP is the difficulty in handling larger use-cases. This was the main rationale behind using the GA. The MILP formalizes also the constraints that have to be respected during the synthesis. The last are preserved by our implementation of the GA.

\subsection{Genetic Algorithm}

Genetic Algorithm is an optimization technique patterned after natural selection in biological evolution. Each possible solution i.e. $\Psi_{i}$ is encoded using a string of bits that we call a chromosome. One or few bits encode a solution for a specific parameter, in our case runnable entity or data signal. Group of bits corresponding to one parameter is called a gene. Later in this paragraph we specify our encoding, generation of initial population and how it is evolved in the consecutive iterations until the stop criteria is met. We also describe the correction mechanism that keeps our population consistent in regard to the constraints.

\subsubsection{Encoding}

Each chromosome $c h_{i}$ represents a specific deployment configuration. Gene $g_{i}$ relates either to runnable entity or a data signal. For the first, gene $g_{i}=c h_{j}\left(r_{k}\right)$ stores the value $V\left(g_{i}\right)$ representing runnable's allocation and partitioning. For a data signal, value stored depends whether it is a global data signal or a data signal that is communicated locally. Value for a global data signal will hold information about the BUS and the message in which it is partitioned. If this is a local data signal $s_{i}$, value depends on whether $s_{i}$ is communicated through the shared resource or no. For the first case, value represents one of the two mechanisms, either $S L$ (value $=1$ ) or $R T$ (value $=2$ ). For the second, value equals 0 .

The gene value $V_{r_{j}}\left(g_{i}\right)$ for the runnable $r_{j}$, this is one number but stores information about the ECU number on which runnable $r_{j}$ is allocated, the task number in which it is partitioned, and the position (order) inside the task. The $V_{r_{j}}\left(g_{i}\right)$ for runnable $r_{j}$ for which selected ECU is $e_{k}$, task $\tau_{l}$ and position $p$ is computed in a specific way, according to (13). The $\max _{E C U}$ is the maximal number of runnables that can be allocated on one ECU and max $_{\text {Task }}$ is the maximal number or runnables that can be partitioned in one task. These values are automatically initialized before running the GA. The $\max _{E C U}$ is computed as a maximal number of runnables that can be hosted by one ECU without violation of utilization (for this WCETs and periods of runnables are used). The max $_{\text {Task }}$ is computed based on the maximal number of runnables with harmonic periods.

The gene value for a data signal, if transmitted on the bus, is computed in a similar way (see (14)). Figure 2 presents an example of a chromosome for a specific deployment configuration.

$$
\begin{aligned}
& V_{r_{j}}\left(g_{i}\right)=(k-1) * \max _{E C U} * \max _{\text {Task }}+(l-1) * \\
& \max _{\text {Task }}+(p-1) \\
& V_{s_{j}}\left(g_{i}\right)=(k-1) * \max _{B U S} * \max _{M s g}+(l-1) * \\
& \max _{M s g}+(p-1)
\end{aligned}
$$

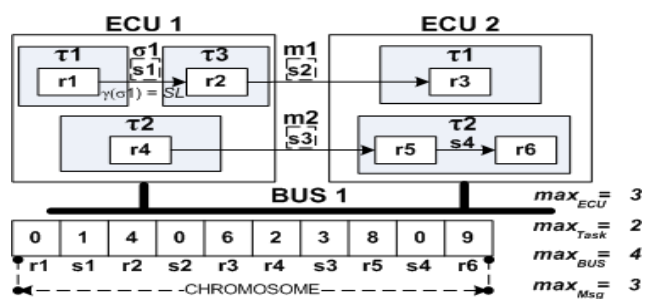
specific configuration 


\subsubsection{Initial Population}

The initial population is generated randomly but to generate correct chromosomes, possible range of values for each gene depends on the values already assigned to others. Correct means chromosome representing deployment configuration that respects the constraints formalized below with the MILP (see subsection 4.2).

\subsubsection{Evolution}

The evolution of a population is through the selection of chromosomes with good fitness and applying the crossover and mutation mechanism on them. The fitness is computed as presented in Section 4. For the selection we are using tournament selector [17] with a tournament size equal to 5 . The crossover operator this is OX3 [18]. It creates two child chromosomes from the two parents. The OX3 choses two random positions in parent chromosomes. Then the values between them are copied from the first/second parent to the second/first child. The rest is copied from the first/second parent to the first/second child. The mutation operator choses a random gene in a chromosome and changes its value to the new random value. The last is selected from the values that don't violate the constraints. In addition it is possible to select the probability for applying the mutation operation. Mutation is done for the child chromosomes resulting from the crossover operation.

\subsubsection{Correction Mechanism}

When generating initial population or applying the mutation operator, the correctness of a new chromosome is preserved. This doesn't hold for the chromosomes resulting from the crossover operation. Therefore on each child chromosome the correction mechanism is called. This is to fix the genes which represent the values violating the constraints.

\subsection{MILP Formulation}

In the MILP formulation, the problem is represented with parameters, decision variables, and constraints over the parameters and decision variables. Moreover, an objective function is defined to characterize the optimal solution.

As we have a large number of parameters, decision variables and constraints, they are directly discussed along with the specific aspects of the problem, in the following sections.

Runnables allocation: Runnables allocation is implicit through the allocation of components. Constraint (15) specifies that each software component is allocated on exactly one ECU. The $\varepsilon\left(s w c_{i}\right)$ is the set of $s w c_{i}$ candidate ECUs. The $a_{s w c_{i}, e_{j}}$ is a binary variable that is 1 if the component $s w c_{i}$ is allocated on ECU $e_{j}$.

$$
\sum_{e_{j} \in \varepsilon\left(s w c_{i}\right)} a_{s w c_{i}, e}=1
$$

Constraint (16) defines the binary variables $x_{s w c_{i}, s w c_{j}, e_{k}}$ based on $a_{s w c_{i}, e_{k}}$ and $a_{s w c_{j}, e_{k}} \cdot x_{s w c_{i}, s w c_{j}, e_{k}}$ is set to 1 if $s w c_{i}$ and $s w c_{j}$ are allocated on the same ECU $e_{k}$, otherwise, $x_{s w c_{i}, s w c_{j}, e_{k}}$ is 0 .

$$
0 \leq a_{s w c_{i}, e_{k}}+a_{s w c_{j}, e_{k}}-2 \cdot x_{s w c_{i}, s w c_{j}, e_{k}} \leq 1
$$

Signals allocation: It is based on the allocation of runnables. Signal is either allocated on none or one bus. In constraint (17), the binary variable $g_{s_{i}}$ indicates if the signal $s_{i}$ is allocated on a bus $\left(g_{s_{i}}=1\right)$ or not $\left(g_{s_{i}}=0\right)$. The binary variable $a_{s_{i} b_{k}}$ indicates if the signal $s_{i}$ is allocated on the bus $b_{k}$. Constraint (17) guarantees that if a signal represents inter-ECU communication, it is allocated on exactly one bus.

$$
\sum_{b_{k} \in \beta} a_{s_{i}, b_{k}}=g_{s_{i}}
$$

Constraint (18) assures that a signal $s_{i}$ is allocated on a bus iff the components of its writer runnables and its reader runnables are allocated on different ECUs.

$$
\begin{gathered}
\forall r_{i} \in r^{w}\left(\zeta^{\prime}\left(s_{i}\right)\right), \quad r_{j} \in \\
r^{r}\left(\zeta^{\prime}\left(s_{i}\right)\right): 1-\sum_{e_{k} \in E} x_{s w c_{(r i)}, s w c_{(r j)}, e_{k}}=g_{s_{i}}
\end{gathered}
$$

Constraint (19) expresses the condition that the signal $s_{i}$ is allocated on the bus $b_{k}$ iff its readers and writers are on the ECUs communicating via $b_{k}$.

$$
\begin{gathered}
\forall_{r_{i} \in r^{w}}\left(\zeta^{\prime}\left(s_{i}\right)\right), r_{j} \in r^{r}\left(\zeta^{\prime}\left(s_{i}\right)\right): 0 \leq \sum_{e_{l} \in E\left(b_{k}\right)} a_{s w c_{r i}, e_{l}}+ \\
\sum_{e_{l} \in E\left(b_{k}\right)} a_{s w c_{r j}, e_{l}}+g_{s_{i}}-3 a_{s_{i}, b_{k}} \leq 2
\end{gathered}
$$

Priority assignment: Constraints on priority assignment are specified in the same way for runnables and signals. Due to the lack of space, we give only the constraints for runnables. Constraint (20) defines the binary variable $\rho_{r i, r j}$ that expresses the priority order between runnables. $\rho_{r i, r j}=1$ means that $r_{i}$ has higher priority than $r_{j}$. If $\rho_{r i, r j}=0$ and $\rho_{r j, r i}=0$ then $r_{i}$ and $r_{j}$ have the same priority order.

$$
\rho_{r i, r j}+\rho_{r j, r i} \leq 1
$$

Constraints (21), (22), (23), (24) and (25) ensure the observance of the symmetric, transitive and inversion properties of the priority order relation.

$$
\begin{gathered}
\rho_{r i, r j}+\rho_{r j, r k}-1 \leq \rho_{r i, r k} \\
\rho_{r i, r j}-\left(\rho_{r j, r k}+\rho_{r k, r j}\right) \leq \rho_{r i, r k} \\
\rho_{r j, r k}-\left(\rho_{r j, r i}+\rho_{r i, r j}\right) \leq \rho_{r i, r k} \\
\rho_{r i, r j}+\rho_{r j, r i}+\rho_{r j, r k}+\rho_{r k, r j} \geq \rho_{r i, r k} \\
\rho_{r i, r j}+\rho_{r j, r i}+\rho_{r j, r k}+\rho_{r k, r j} \geq \rho_{r k, r i}
\end{gathered}
$$

Runnables with non-harmonic periods are not allowed to have the same priority order. This is represented by the constraint (26). If $\left(P_{r i} \geq P_{r j}\right)$ and $\left(P_{r i} \bmod P_{r j} \neq 0\right)$ :

$$
1=\rho_{r j, r i}+\rho_{r i, r j}
$$


Runnables sequence order: A total order is defined for runnables to express the execution sequence order for runnables with the same priority. Constraint (27) defines the binary variable $S o_{r i, r j}$ that represents the sequence order between $r_{i}$ and $r_{j}$. The sequence order is total, i.e. either $r_{i}$ is executed before $r_{j}\left(S o_{r i, r j}=\right.$ 1) or $r_{j}$ before $r_{i}\left(S o_{r j, r i}=1\right)$.

$$
S o_{r i, r j}+S o_{r j, r i}=1
$$

Constraint (28) guarantees the antisymmetric and transitive properties of the sequence order relation.

$$
S o_{r i, r j}+S o_{r j, r k}-1 \leq S o_{r i, r k}
$$

Dependency constraints: Dependencies between runnables allow to set some sequence and priority orders i.e. when the execution of $r_{j}$ depends on the execution of $r_{i}$, i.e. $\left(r_{i} \rightarrow r_{j}\right)$, it doesn't make sense to give higher priority or sequence to $r_{i}(29)$.

$$
\text { if }\left(r_{i} \rightarrow r_{j}\right): \rho_{r j, r i}=0 \text { and } S o_{r j, r i}=0
$$

To express next constraints we are defining the following set of binary variables: $S n H p_{r i, r j, e_{k}}=$ 1 indicates that $r_{i}$ and $r_{j}$ are allocated on the same ECU $e_{k}$ and $r_{j}$ has higher priority than $r_{i}(30)$.

$$
0 \leq \rho_{r j, r i}+x_{s w c_{(r i)}, s w c_{(r j)}, e_{k}}-2 S n H p_{r i, r j, e_{k}} \leq 1
$$

$S n D p_{r i, r j, e_{k}}=1$ determines that $r_{i}$ and $r_{j}$ are allocated on the same ECU $e_{k}$ but they have different priority order (31).

$$
S n D p_{r i, r j, e_{k}}=S n H p_{r i, r j, e_{k}}+S n H p_{r j, r i, e_{k}}
$$

$S n S p_{r i, r j, e_{k}}=1$ indicates that $r_{i}$ and $r_{j}$ have the same priority and they reside on the same ECU $e_{k}$ (32).

$$
S n S p_{r i, r j, e_{k}}+S n D p_{r i, r j, e_{k}}=x_{s w c_{(r i)}, s w c_{(r j)}, e_{k}}
$$

Protection mechanism: Communication variables in inter-tasks communication within an ECU are either protected by RT blocks or semaphore locks. Constraints (33) and (34) determine the binary variable $Y_{\sigma i}$ that says if the shared resource $\sigma_{i}$ should be protected $\left(Y_{\sigma i}=1\right)$ or not $\left(Y_{\sigma i}=0\right)$. When all writer and reader runnables of a shared resource are on the same ECU and task (i.e. they have the same priority), the protection of the shared resource is not needed (33). Within the same ECU, if there is at least one reader runnable with different priority as one of the writer runnables, the shared resource needs to be protected (34).

$$
\begin{aligned}
& Y_{\sigma i} \leq \sum_{r_{i} \in r^{w}\left(\sigma_{i}\right)} \quad \sum_{r_{j} \in r^{r}\left(\sigma_{i}\right)} \quad \sum_{e_{k} \in E} S n D p_{r i, r j, e_{k}} \\
& \forall r_{i} \in r^{w}\left(\sigma_{i}\right), r_{j} \in r^{r}\left(\sigma_{i}\right): Y_{\sigma_{i}} \geq \sum_{e_{k} \in E} S n D p_{r i, r j, e_{k}}
\end{aligned}
$$

To specify the mechanism of protection, we define two binary variables mem $_{\sigma i}$ and $\operatorname{lock}_{\sigma i} \cdot$ mem $_{\sigma i}=1$ indicates that $\sigma_{i}$ is protected using RT blocks and $\operatorname{lock}_{\sigma i}=1$ that the protection mechanism for $\sigma_{i}$ is the semaphore lock. Constraint (35) gives the relationship between variables $Y_{\sigma i}, m_{e} m_{\sigma i}$ and lock $_{\sigma i}$.

$$
Y_{\sigma i}=\operatorname{mem}_{\sigma i}+\operatorname{lock}_{\sigma i}
$$

Memory utilization: The additional memory cost in the MILP is defined using some variables. We define the binary variable $V_{\sigma i}$ based on the priorities of writer and readers runnables. The $V_{\sigma i}=0$ means that for the shared resource $\sigma_{i}$ there are no reader runnables with higher priority than the writer, the value of $V_{\sigma i}$ in this case is fixed by constraint (36). If there is at least one reader runnable with higher priority than the writer runnable then the value of $V_{\sigma i}$ is set to 1 in (37).

$\forall r_{i} \in r^{w}\left(\sigma_{i}\right): V_{\sigma_{i}} \leq \sum_{r_{j} \in r^{r}\left(\sigma_{i}\right)} \sum_{e_{k} \in E} S n H p_{r_{i}, r_{j}, e_{k}}$
$\forall r_{i} \in r^{w}\left(\sigma_{i}\right), r_{j} \in r^{r}\left(\sigma_{i}\right): \sum_{e_{k} \in E} S n H p_{r_{i}, r_{j}, e_{k}} \leq V_{\sigma_{i}}$

The memory needed for each shared resource is computed as in constraint (38), where, $Z_{r_{i}, r_{j}, e_{k}}$ is a binary variable equal to $\operatorname{SnHp}_{r_{i}, r_{i}, e_{k}} * \operatorname{mem}_{\sigma_{i}}$. $Z_{\sigma_{i}}^{\prime}$ is another binary variable set to 1 if both $V_{\sigma i}$ and $m^{2} m_{\sigma_{i}}$ are equal to 1 , otherwise, it is equal to 0 . $Z_{r_{i}, r_{j}, e_{k}}$ and $Z_{\sigma_{i}}^{\prime}$ are defined in the same way as the variable $S n H p_{r i, r j, e_{k}}$ in constraint (30).

$$
\begin{array}{r}
\forall r_{i} \in r^{w}\left(\sigma_{i}\right): \text { memSize }_{\sigma i}= \\
\sum_{r j \in \mathrm{r}^{r}\left(\sigma_{i}\right)} \sum_{e_{k} \in E} Z_{r_{i}, r_{j}, e_{k}}+\operatorname{mem}_{\sigma_{i}}+Z_{\sigma_{i}}^{\prime}
\end{array}
$$

Semaphore lock: Memory consumption can be avoided by using semaphore locks. However, this will result in a blocking time for runnables (according to PCP) equal to the largest critical section of lower priority runnables sharing resources with higher or equal priority ceiling. Constraint (39) expresses the blocking time of $r_{i}$ based on the binary variable cond $_{r i, r j, \sigma i}$, which represents the necessary condition to consider $r_{j}$ during the computation of $B_{r i}$. The definition of this condition is given in constraint (40). It consists in the combination of three sub conditions: i) $r_{j}$ is lower priority than $r_{i}$, ii) $\sigma_{i}$ is a shared variable protected by a lock and iii) the priority ceiling of $\sigma_{i}$ is higher or equal to the priority of $r_{i}$. The third condition is expressed by the binary variable $P C_{r i, \sigma i}$.

$$
\begin{gathered}
B_{r i} \geq \operatorname{cond}_{r i, r j, \sigma i} * \omega_{p_{r_{j}, \sigma i}} \\
0 \leq P C_{r i, \sigma i}+\operatorname{lock}_{\sigma i}+\sum_{e_{k} \in E} \operatorname{SnH}_{r i, r j, e_{k}} \\
+-3 \operatorname{cond}_{r i, r j, \sigma i} \leq 2
\end{gathered}
$$

As priority of runnables sharing directly a resource is always lower or equal to the priority ceiling of this resource, $\forall r_{i} \in\left(r^{w}\left(\sigma_{i}\right) \cup r^{r}\left(\sigma_{i}\right)\right): P C_{r i, \sigma i}=$ lock $_{\sigma i}$.

For runnables $r_{i}$ that do not share the resource $\sigma_{i}$, the value of $P C_{r i, \sigma i}$ depends on the priority of all runnables $r_{j}$ sharing $\sigma_{i}$ i.e. if there is at least one runnable $r_{j}$ sharing $\sigma_{i}$ with higher or equal priority than $r_{i}, P C_{r_{i}, \sigma_{i}}=1$ (41) otherwise if all runnables $r_{j}$ sharing $\sigma_{i}$ have lower priority than $r_{i}$ then $P C_{r i, \sigma i}=0$ (42). 


$$
\begin{gathered}
\forall_{r_{i} \notin\left(r^{w}\left(\sigma_{i}\right) \cup r^{r}\left(\sigma_{i}\right)\right)} \text { and } \forall_{r_{j} \in\left(r^{w}\left(\sigma_{i}\right) \cup r^{r}\left(\sigma_{i}\right)\right)}: P C_{r_{i}, \sigma_{i}} \geq \\
\sum_{e_{k} \in E} S n S p_{r i, r j, e_{k}}+\sum_{e_{k} \in E} \operatorname{SnHp}_{r i, r j, e_{k}} \\
\forall_{r_{i} \notin\left(r^{w}\left(\sigma_{i}\right) \cup r^{r}\left(\sigma_{i}\right)\right)} P C_{r_{i}, \sigma_{i}} \leq \\
\sum_{r_{j} \in\left(r^{w}\left(\sigma_{i}\right) \cup r^{r}\left(\sigma_{i}\right)\right)}\left(1-\sum_{e_{k} \in E} \operatorname{SnH}_{r i, r j, e_{k}}\right)
\end{gathered}
$$

Worst-case response time computation: The computation of runnables WCRT needs the definition of some variables, $S n H o_{r i, r j, e_{k}}, V_{r i, r j}$ and $I_{r i, r j, e_{k}}$. The binary variable $\mathrm{SnHo}_{r i, r j, e_{k}}$ indicates whether the runnable $r_{j}$, that is allocated on the same ECU as $r_{i}$ and has the same priority as $r_{i}$, has a higher execution order than $r_{i}$. The definition of the integer variable $V_{r_{i}, r_{j}}$, which represents the number of possible interferences of $r_{j}$ on $r_{i}\left(V_{r_{i}, r_{j}}=\left\lceil\frac{R_{r_{i}}}{P_{r_{j}}}\right\rceil\right)$, is captured by
(43).

$$
0 \leq V_{r_{i}, r_{j}}-\left(R_{r_{i}} / P_{r_{j}}\right) \leq 1
$$

The integer variable $I_{r i, r j, e_{k}}$ is the number of possible interferences of $r_{j}$ on $r_{i}$ when $r_{j}$ is higher priority than $r_{i}\left(I_{r i, r j, e_{k}}=V_{r_{i}, r_{j}} * \rho_{r j, r i}\right)$. We use in the same way as previous the Big $\mathrm{M}$ method to linearize $I_{r i, r j, e_{k}}$. Finally, (44) gives the computation of WCRT.

$\sum_{r_{j}} \sum_{e_{k} \in E} \mathrm{SnHo}_{r i, r j, e_{k}} \cdot C_{r j, e_{k}}+\sum_{r_{j}} \sum_{e_{k} \in E} I_{r i, r j, e_{k}} \cdot C_{r j, e_{k}}$

$+B_{r_{i}}+\sum_{e_{k} \in E} a_{s w c\left(r_{i}\right), e_{k}} \cdot C_{r_{i}, e_{k}}=R_{r_{i}}$

The computation of signals WCRT is similar to runnables with the only difference that the blocking time $B_{s_{j}}$ is computed as the largest WCTT of any group of equal priority signals sharing the same bus (45).

$$
\begin{array}{r}
\forall_{s_{j} \neq s_{i}}: B_{s_{i}} \geq \sum_{b_{k} \in \beta} \operatorname{SnD} p_{s_{i}, s_{j}, b_{k}} \cdot C_{s_{j}, b_{k}}+ \\
\sum_{s_{l}} \sum_{b_{k} \in \beta} \operatorname{SnSp} p_{s_{j}, s_{l}, b_{k}} \cdot C_{s_{l}, b_{k} .} \operatorname{SnDp} p_{s_{i}, s_{l}, b_{k}}
\end{array}
$$

\section{Experiments}

There are two goals of the conducted experiments. The first one is to show the quality of results obtained with the GA (with respect to the optimal solutions given by the MILP) and the runtimes. Next, we compare our technique with the existing approaches that do not consider partitioning. The last has a high influence when optimizing the timing responses and the memory overhead.

\subsection{GA vs MILP}

In order to assess the quality of results obtained with the GA we compared it to the MILP. MILP assures optimality of the result in case if the solver finishes with no error. Unfortunately for the larger use-cases, solver that we used - CPLEX [19], although run on a powerful machine, is not able to provide an optimal result. It either finishes computation with an error message not providing any result or it stops with an out of memory message. In the second case, it returns a result, however it is not sure whether it is optimal. Therefore we have set use-cases for which we can infer optimal configurations (solutions). This has been done by first applying the weight 1 for the function responsible for optimizing end-to-end responses and fixing a simple use-case shown in the

Figure $3^{2}$. For this case, with the only optimization of response time, the set of optimal configurations contains any possible partitioning, and for each shared resource (if any) the protection mechanism is the RT. The left configuration presented in Figure 4 is an example of an optimal solution for the simple use-case.

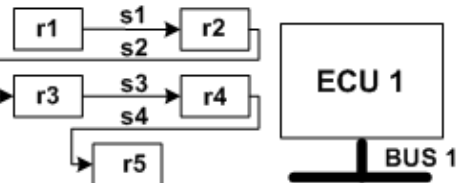

Figure 3 Non-replicated Use-Case

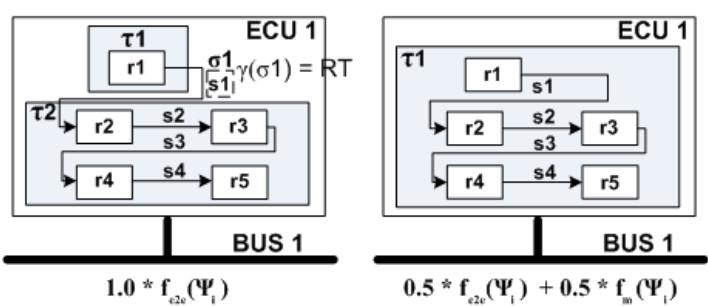

Figure 4 Optimal configurations

Once the simple use-case has been fixed, other usecases were found by replicating the simple use-case. This means that, each path, runnable, ECU and BUS is replicated. Hence when replicating by 11 we obtained a use-case with 55 runnables, 11 ECUs and 11 BUS-es. Also, we connected each ECU to the original BUS and all the replicas. Let us remark that for the replicated use-cases, the set of optimal configurations is characterized by having each ECU containing only one path (no inter-ECU communication). Figure 5 shows the runtime for the MILP and the GA. Indexes on the horizontal axe express the factor for the replication. As can be seen, MILP on average gives the results in shorter time. However the Figure 6 shows that when architecture has been multiplied 6, 9, 10 and 11 times, the solver didn't return any solution. This was due to the returned error. For the factor 5, 7 and 8, the CPLEX finished execution with "out of memory exception". Nevertheless for the factor 5, returned result is optimal, which is not the case for 7 and 8 . The GA for all the replication factors was able to return the optimal solution. We run similar tests but with weights 0.5 for the end-to-end responses and 0.5 for the memory optimization. Set of the optimal solutions for the nonreplicated use case contains only one configuration in which all the runnables are partitioned in one task.

\footnotetext{
2 For sake of simplicity, we omitted from the image the presentation of software components. In this case we assume one software component per runnable entity.
} 


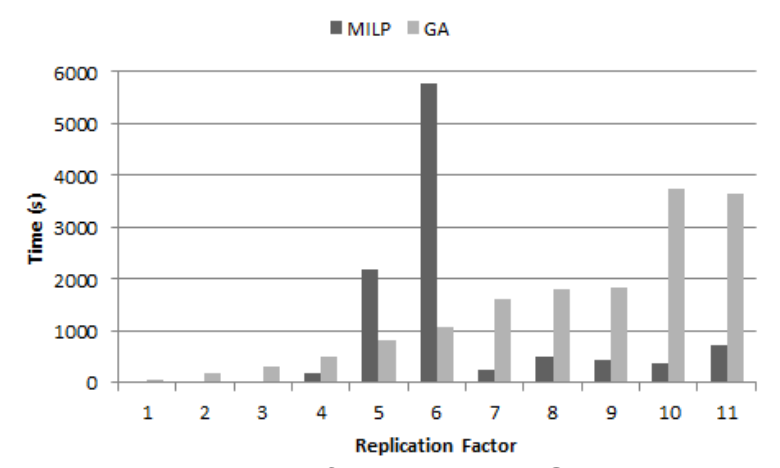

Figure 5: Runtime for MILP and GA (1.0* $\left.\mathbf{f}_{e 2 e}\left(\Psi_{i}\right)\right)$

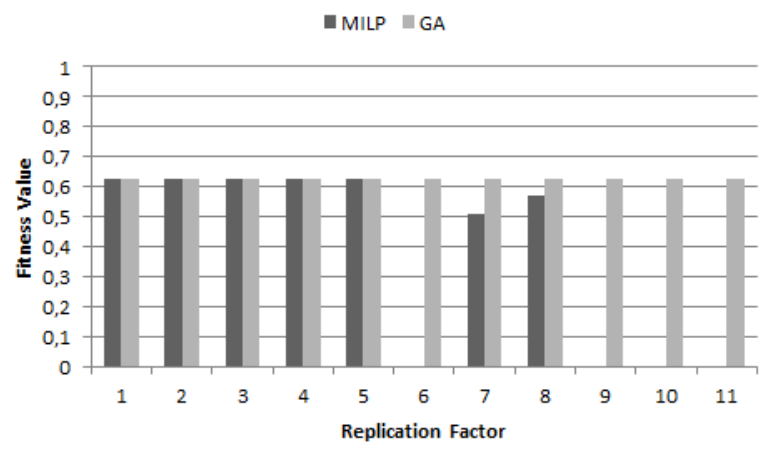

Figure 6: Results for MILP and GA (1.0 * $\left.\mathbf{f}_{e 2 e}\left(\Psi_{i}\right)\right)$

For the replicated use-cases, each ECU must contain only one path. In this case, on average, MILP provides the results in a shorter time (see Figure 7). However, already for the replication factor 5 (see Figure 8) the returned result was not optimal and starting from 9, CPLEX didn't provide any result. The degradation of the results given by the GA, started from factor 8 . In general, the reason for this is that when using equal weights for the latency and memory optimization functions, the set of optimal configurations is smaller than if optimizing only end-to-end latencies. This is due to the fact that optimal solutions only have the runnables of the same path partitioned in the same task. The configuration on the right side of the Figure 4 represents the only optimal configuration for the nonreplicated use-case. In all of those use-cases the GA was run with an initial population of 10000 . The algorithm stops if during the 20 consecutive evolutions, the fittest chromosome doesn't change. When we run the GA on the population of 100000 we were able to obtain the optimal solution for the second metric and the replication from 8 to 11 . This however increased the runtime to around 12 hours on a $2.4 \mathrm{GHz}$ single processor computer with $8 \mathrm{~GB}$ of memory.

\subsection{Evaluation against approaches with none or partial Partitioning}

This set of tests shows the added value of considering the partitioning. Therefore we will compare the results obtained with the GA with those that doesn't consider

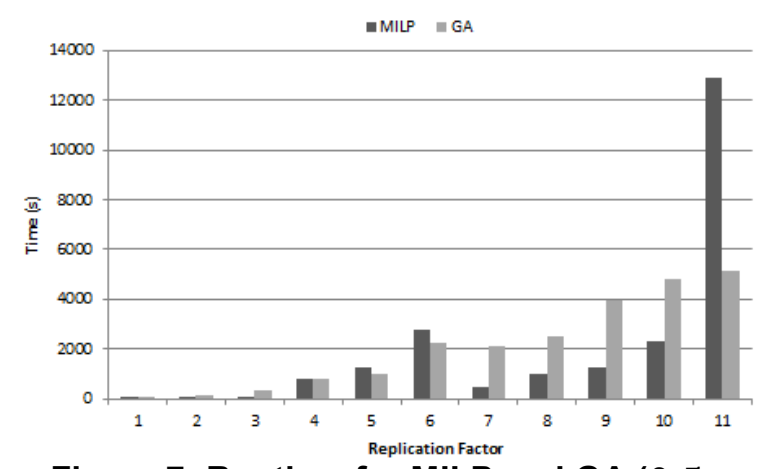

Figure 7: Runtime for MILP and GA (0.5 * $\left.\mathbf{f}_{e 2 e}\left(\boldsymbol{\Psi}_{i}\right)+0.5 * \mathbf{f}_{m}\left(\Psi_{i}\right)\right)$

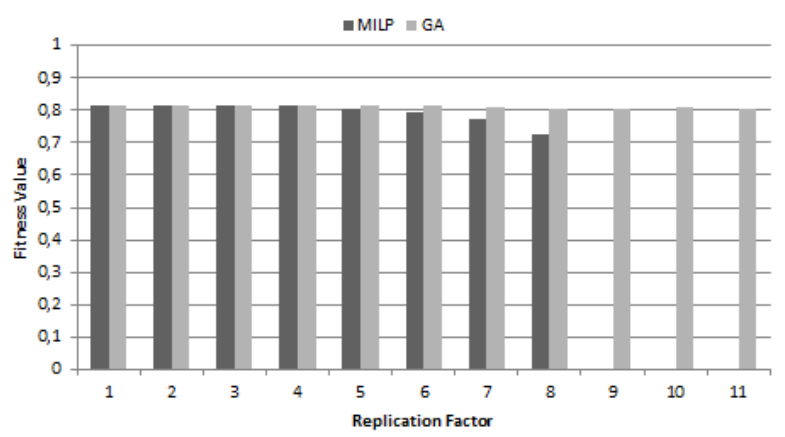

Figure 8: Results for MILP and GA (0.5 *

$$
\left.\mathbf{f}_{e 2 e}\left(\Psi_{i}\right)+0.5 * \mathbf{f}_{m}\left(\Psi_{i}\right)\right)
$$

the partitioning (which is the case for [8]) or approaches that consider only partial partitioning, i.e. only runnables of the same period can be merged together (the case for [10]). The last two were implemented in MILP hence for them the obtained results are optimal in case when solver returned the result without error. The tests were run on a set of random input architectures.

Figure 9 and 10 show that consideration of the partitioning has an impact on the optimization metrics. For the fitness 1.0 for the $\mathrm{f}_{e 2 e}\left(\Psi_{i}\right)$ (Figure 9) the GA obtained results $34,87 \%$ better than those with no partitioning and $16,48 \%$ from those with partial partitioning. Please note that for all of the approaches the same schedulability test is used (see subsection 3.3). Hence the metric improvement is a consequence of the constrained design space for the approach with partial and no partitioning. Possible, further improvement is to change the way in which $C_{\tau_{i, j}}$ from the equation 6 is computed for the approach with full partitioning. For the moment it has pessimistic assumption that all the runnables, even those with harmonic but not the same periods will always be activated.

Considering Figure 10, the results of the GA were $6.8 \%$ better than those obtained with the approach disregarding partitioning, and $5.7 \%$ better from those which limit partitioning to the same periods. Let us note that for 35 runnables, CPLEX didn't return any result. 


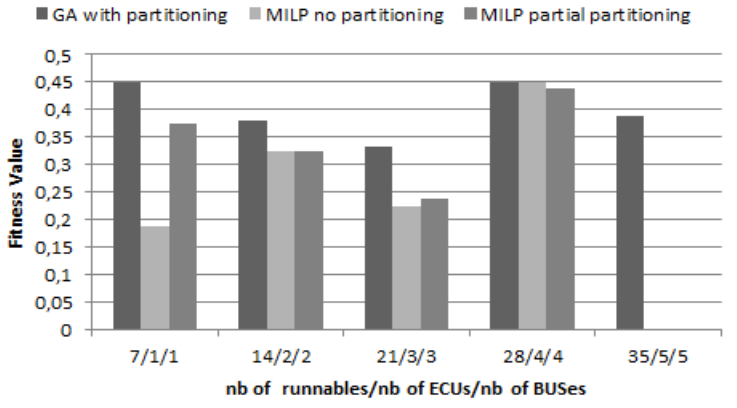

Figure 9: Comparison using fitness function $\left(1.0 * \mathbf{f}_{e 2 e}\left(\Psi_{i}\right)\right)$

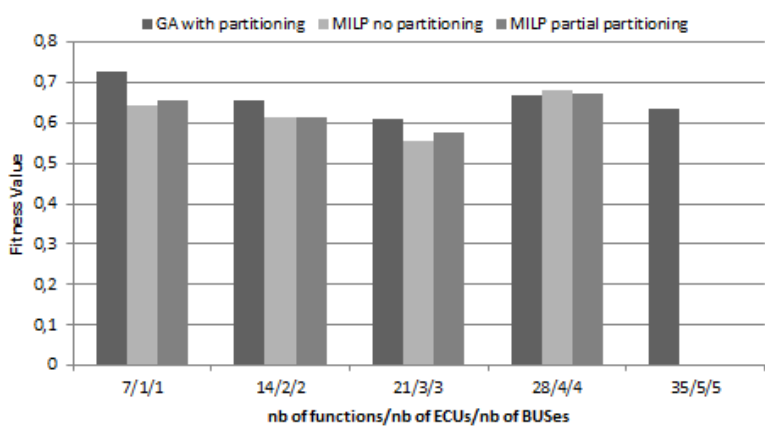

Figure 10: Comparison using fitness function

$\left(0.5 * \mathbf{f}_{e 2 e}\left(\Psi_{i}\right)+0.5 * \mathbf{f}_{m}\left(\Psi_{i}\right)\right)$

\section{Conclusions and Future Work}

We presented a method for the optimized synthesis of the AUTOSAR compliant architectures. We proposed two techniques, MILP and GA. The main contribution of this work lies in a holistic approach that considers allocation, partitioning, scheduling and ordering together. As shown in the experimental part, employment of partitioning that is neglected in the current works, improves the optimization metrics. In addition we have evaluated our heuristic, i.e. the GA against MILP (i.e. exact approach) in terms of runtimes and the quality of results. As a future work we envisage to further scale our technique by provision of the parallelism in the implementation of the GA.

\section{References}

[1] AUTOSAR Methodology, AUTOSAR Std. http://www.autosar.org/download/-

AUTOSARMethodology.pdf

[2] AUTOSAR Specification of Timing Extensions, AUTOSAR Std. http://www.autosar.org/download/R4.0/AUTOSAR_TPS_TimingExtensions.pdf

[3] I. Bate and P. Emberson, "Incorporating scenarios and heuristics to improve flexibility in real-time embedded systems," in Proceedings of the 12th IEEE Real-Time and Embedded Technology and Applications Symposium, 2006, pp. 221-230.

[4] W. Peng, H. Li, M. Yao, and Z. Sun, "Deployment optimization for autosar system configuration," in
Computer Engineering and Technology (ICCET), 2010.

[5] R. Long, H. Li, W. Peng, Y. Zhang, and M. Zhao, "An approach to optimize intra-ecu communication based on mapping of autosar runnable entities," in ICESS, 2009.

[6] R. Racu, M. Jersak, and R. Ernst, "Applying sensitivity analysis in real-time distributed systems," in Proceedings of the 11th IEEE Real Time on Embedded Technology and Applications Symposium, 2005, pp. 160-169.

[7] Q. Zhu, H. Zeng, W. Zheng, M. D. Natale, and A. Sangiovanni-Vincentelli, "Optimization of task allocation and priority assignment in hard real-time distributed systems," ACM Transactions on Embedded Computing Systems, vol.11, no. 4, pp. 85:1-85:30, 2012.

[8] Q. Zhu, Y. Yang, E. Scholte, M. Di Natale, and A. Sangiovanni-Vincentelli, "Optimizing extensibility in hard real-time distributed systems," in Proceedings of the 15th IEEE Real-Time and Embedded Technology and Applications Symposium. IEEE, 2009, pp. 275284.

[9] A. Ferrari, M. D. Natale, G. Gentile, G. Reggiani, and P. Gai, "Time and memory tradeoffs in the implementation of autosar components," in DATE, 2009, pp. 864-869.

[10] M. Zhang and Z. Gu, "Optimization issues in mapping autosar components to distributed multithreaded implementations," in International Symposium on Rapid System Prototyping, 2011, pp. 23-29.

[11] H. Zeng and M. D. Natale, "Efficient implementation of autosar components with minimal memory usage," in SIES, 2012, pp. 130-137.

[12] H. Zeng, M. D. Natale, and Q. Zhu, "Optimizing stack memory requirements for real-time embedded applications," in ETFA, 2012, pp. 1-8.

[13] A. Aleti, B. Buhnova, L. Grunske, A. Koziolek, and I. Meedeniya, "Software architecture optimization methods: A systematic literature review," IEEE Transactions on Software Engineering, To appear.

[14] "Autosar system template," http://www.autosar.org/download/R4.0/AUTOSAR_TPS_SystemTemplate.pdf

[15] The Mathworks Simulink and StateFlow User's Manuals. http://www.mathworks.com

[16] L. Sha, R. Rajkumar, and J. P. Lehoczky, "Priority inheritance protocols: An approach to real-time synchronization," IEEE Trans. Computers, vol. 39, no. 9, 1990.

[17] B. L. Miller, B. L. Miller, D. E. Goldberg, and D. E. Goldberg, "Genetic algorithms, tournament selection, and the effects of noise," Complex Systems, vol. 9, pp. 193-212, 1995.

[18] L. Davis, Ed., Handbook of Genetic Algorithms. Van Nostrand Reinhold, 1991.

[19] http://www-01.ibm.com/software/integration/optimization/cplex-optimizer/. 